\title{
AN ALGEBRAIC-METRIC EQUIVALENCE RELATION OVER $p$-ADIC FIELDS
}

\author{
MARIAN VÂJÂITU \\ Simion Stoilow Institute of Mathematics of the Romanian Academy, Research Unit 5, \\ P. O. Box 1-764, RO-014700 Bucharest, Romania \\ e-mail: Marian.Vajaitu@imar.ro \\ and ALEXANDRU ZAHARESCU \\ Department of Mathematics, University of Illinois at Urbana-Champaign, 1409 W. Green Street, \\ Urbana, IL 61801, USA \\ e-mail: zaharesc@math.uiuc.edu
}

(Received 24 November 2011; revised 13 March 2012; accepted 17 April 2012)

\begin{abstract}
Let $p$ be a prime number, $\mathbf{Q}_{p}$ the field of $p$-adic numbers, $K$ a finite field extension of $\mathbf{Q}_{p}, \bar{K}$ a fixed algebraic closure of $K$ and $\mathbf{C}_{p}$ the completion of $\bar{K}$ with respect to the $p$-adic valuation. We introduce and investigate an equivalence relation on $\mathbf{C}_{p}$, defined in terms of field extensions and metric properties of Galois orbits over $K$.
\end{abstract}

2000 Mathematics Subject Classification. 11S99.

1. Introduction. Let $p$ be a prime number, $\mathbf{Q}_{p}$ the field of $p$-adic numbers, $K$ a fixed finite field extension of $\mathbf{Q}_{p}, \bar{K}$ a fixed algebraic closure of $K$ and $\mathbf{C}_{p}$ the completion of $\bar{K}$ with respect to the $p$-adic valuation. Here and in what follows we denote by $|$.$| the$ $p$-adic absolute value on $\mathbf{C}_{p}$, normalised by $|p|=\frac{1}{p}$. We also denote by $O_{\mathbf{C}_{p}}$ the ring of integers of $\mathbf{C}_{p}$ and by $G_{K}$ the group of continuous automorphisms of $\mathbf{C}_{p}$ over $K$. The group $G_{K}$ is canonically isomorphic to the Galois group $G a l(\bar{K} / K)$, see [1], [2] and [6]. By the Galois orbit over $K$ we mean a set of the form $C_{K}(T):=\left\{\sigma(T): \sigma \in G_{K}\right\}$, with $T$ in $\mathbf{C}_{p}$. Associated to each such Galois orbit $C_{K}(T)$, we have the Haar distribution (in the sense of Mazur and Swinnerton-Dyer [7]) on $C_{K}(T)$, call it $\pi_{K, T}$, which is a unique distribution on $C_{K}(T)$ with values in $\mathbf{Q}_{p}$, normalised by $\pi_{K, T}\left(C_{K}(T)\right)=1$, which is $G_{K}$-invariant, in the sense that for any ball $B$ in $C_{K}(T)$ and for any $\sigma \in G_{K}$ one has $\pi_{K, T}(\sigma(B))=\pi_{K, T}(B)$.

Our goal in this short paper is to introduce an algebraic-metric equivalence relation on $\mathbf{C}_{p}$, which appears to have some nice properties. We establish a few of them in the present paper. This equivalence relation is defined in terms of field extensions of $K$ and metric properties of Galois orbits over $K$. Our starting point is to consider the following natural question: Given two elements $T$ and $U$ of $\mathbf{C}_{p}$, under which circumstances is there a canonical way to define a map from the Galois orbit $C_{K}(T)$ to the Galois orbit $C_{K}(U)$ ? The existence of such a map would have useful implications in questions related to integration along Galois orbits with respect to the Haar distribution, and to the problem of the existence of trace over $K$ of transcendental elements over $K$ (see [3], [5] and [11]). Given two elements $T$ and $U$ of $\mathbf{C}_{p}$, the obvious choice for the definition of a natural map from $C_{K}(T)$ to $C_{K}(U)$ would be to take each element $z$ in $C_{K}(T)$, write 
it in the form $z=\sigma(T)$ with $\sigma \in G_{K}$ and then send it to the element $\sigma(U)$ of $C_{K}(U)$. In general, this is not a well-defined map. In order for this map to be well defined, the following condition is necessary and sufficient: For any $\sigma \in G_{K}$ for which $\sigma(T)=T$, one also has $\sigma(U)=U$. The automorphisms $\sigma \in G_{K}$ that satisfy the equality $\sigma(T)=T$ form a closed subgroup of $G_{K}$, call it $H_{K, T}$, and similarly the automorphisms satisfying $\sigma(U)=U$ form a closed subgroup $H_{K, U}$ of $G_{K}$. The above condition then asks for the inclusion of $H_{K, T} \subseteq H_{K, U}$. If this condition holds, then one has a well-defined map from $C_{K}(T)$ to $C_{K}(U)$ given by $\sigma(T) \mapsto \sigma(U), \sigma \in G_{K}$. We denote this map by $h_{K, T, U}$. If the other inclusion also holds so that we have equality, $H_{K, T}=H_{K, U}$, then we also have a map $h_{K, U, T}: C_{K}(U) \rightarrow \mathbf{C}_{K}(T)$ given by $\sigma(U) \mapsto \sigma(T)$, and the two maps, $h_{K, T, U}$ and $h_{K, U, T}$, are bijections, inverse to each other. By the Galois theory in $\mathbf{C}_{p}$, as developed by Tate [9], Sen [8] and Ax [4], we know that the closed subgroups of the Galois group $G_{K}$ are in one-to-one correspondence with the closed subfields of $\mathbf{C}_{p}$, which contain $K$. Therefore, if we denote by $E_{K, T}$ and $E_{K, U}$ the topological closure of the fields $K(T)$ and respectively $K(U)$ in $\mathbf{C}_{p}$, the above condition $H_{K, T}=H_{K, U}$ is equivalent to the equality $E_{K, T}=E_{K, U}$. Let us assume now that the elements $T$ and $U$ of $\mathbf{C}_{p}$ are such that $E_{K, T}=E_{K, U}$, and consider the maps $h_{K, T, U}$ and $h_{K, U, T}$. These maps then may be used to transfer metric information between the Galois orbits $C_{K}(T)$ and $C_{K}(U)$. The best case, in terms of the accuracy of this transfer of metric information, is when the maps $h_{K, T, U}$ and $h_{K, U, T}$ are isometries. We take this as our definition of equivalence. Thus, given two elements $T$ and $U$ of $\mathbf{C}_{p}$, we say that these are equivalent over $K$, and write $T \sim_{K} U$ if and only if $E_{K, T}=E_{K, U}$ and the maps $h_{K, T, U}$ and $h_{K, U, T}$ are isometries. It is easy to see that $\sim_{K}$ is an equivalence relation on $\mathbf{C}_{p}$. Let us remark that in case $T$ and $U$ are algebraic over $K$, the equality $E_{K, T}=E_{K, U}$ reduces to the equality $K(T)=K(U)$.

In what follows, we establish some properties of the equivalence relation $\sim_{K}$ defined above. We focus on three basic questions. The first one asks whether there is any connection between this equivalence relation and the metric symbol introduced and studied in [10]. The answer is that there is such a connection. This connection provides additional motivation for the study of the equivalence relation $\sim_{K}$, as this equivalence relation is suitable for addressing phenomena in $\mathbf{C}_{p}$ that are not expressible in terms of the original metric symbol from [10]. The second question is whether there are any simple transformations that behave nicely with respect to the equivalence relation $\sim_{K}$, in the sense that many elements $T$ of $\mathbf{C}_{p}$ are sent to elements equivalent to themselves. We will see that fractional linear transformations of $\mathbf{P}^{1}\left(\mathbf{C}_{p}\right)=\mathbf{C}_{p} \cup\{\infty\}, z \rightarrow \frac{a z+b}{c z+d}$, with $a, b, c, d \in K$, send large classes of elements $T$ of $\mathbf{C}_{p}$ to elements equivalent to themselves. The third problem is to investigate the topological properties of the set of elements equivalent to a given element $T$ of $\mathbf{C}_{p}$. We prove that all the equivalence classes are closed in $\mathbf{C}_{p}$. The results mentioned above show that this equivalence relation enjoys some nice properties, and deserves further study.

2. Connection with the metric symbol. In [10] a metric symbol was defined for pairs of polynomials. Precisely, one considers pairs of monic polynomials $f(X), g(X) \in$ $K[X]$ of degree a prime number $q$. For such a pair one defines a metric symbol $\left(\frac{g}{f}\right)$ by the following rule:

$$
\left(\frac{g}{f}\right)=\left\{\begin{array}{ll}
1 & \text { if } v(R(f, g))>\frac{q}{q-1} v(\Delta(f)) \\
-1 & \text { else }
\end{array},\right.
$$


where $\Delta(f)$ denotes the discriminant of $f, R(f, g)$ denotes the resultant of $f$ and $g$ and $v$ denotes the $p$-adic valuation. Although the above definition is not symmetric in $f$ and $g$, this metric symbol has some nice properties. One has the following result.

THEOREM 1. ([10]). (i) (Irreducibility criterion): If $f$ is irreducible and $\left(\frac{g}{f}\right)=1$ then $g$ is also irreducible.

(ii) (Transitivity): Iff is irreducible and $\left(\frac{g}{f}\right)=\left(\frac{h}{g}\right)=1$, then $\left(\frac{h}{f}\right)=1$.

(iii) (Reciprocity Law): Iff and $g$ are irreducible then

$$
\left(\frac{g}{f}\right)=\left(\frac{f}{g}\right)
$$

We also need the following lemma (see for example [10]).

Lemma 1. Let $f \in K[X]$ be irreducible of prime degree $q$. Then the distance between any two distinct roots off is the same.

Let now $q$ be a prime number and let $f(X)$ and $g(X)$ be monic polynomials with coefficients in $K$ of degree $q$, irreducible over $K$, such that $\left(\frac{f}{g}\right)=1$. Then for any root $\alpha$ of $f(X)$, we claim that there is a unique root $\beta$ of $g(X)$ in $\bar{K}$, which satisfies the equality

$$
|\alpha-\beta|=\min \{|\alpha-\theta|: g(\theta)=0\} .
$$

Indeed, let us assume that the above equality holds for two distinct roots $\beta$ and $\beta^{\prime}$ of $g(X)$. Then from $|\alpha-\beta|=\left|\alpha-\beta^{\prime}\right|$ it follows that $\left|\beta-\beta^{\prime}\right| \leq|\alpha-\beta|$. By the above lemma, the distance between any two roots of $g(X)$ is the same, so all these distances are bounded by $|\alpha-\beta|$. On the other hand, the distance between any root of $f(X)$ and any root of $g(X)$ is at least $|\alpha-\beta|$. Indeed, via a suitable automorphism of the Galois group each such distance coincides with the distance from $\alpha$ to a root of $g(X)$, which in turn is at least $|\alpha-\beta|$. Therefore, the geometric mean of the distances between the roots of $g(X)$ is less than or equal to the geometric mean of the distances between the roots of $f(X)$ and the roots of $g(X)$. By expressing the above two geometric means in terms of the discriminant of $g(X)$ and respectively the resultant of $f(X)$ and $g(X)$, one sees that the above is in contradiction with our assumption that $\left(\frac{f}{g}\right)=1$. Therefore, the above $\beta$ is unique, as claimed.

We then have $\alpha \sim_{K} \beta$. Indeed, let $\left\{\alpha=\alpha_{1}, \alpha_{2}, \ldots, \alpha_{q}\right\}$ and $\left\{\beta=\beta_{1}, \beta_{2}, \ldots, \beta_{q}\right\}$ be all the distinct roots of $f$ and $g$ in $\bar{K}$. We can arrange the set of all distinct roots of $g$ such that

$$
|\alpha-\beta|=\left|\alpha_{i}-\beta_{i}\right|=\min \left\{\left|\alpha_{i}-\theta\right|: g(\theta)=0\right\} .
$$

By (1) and Theorem 1 one has

$$
v(R(f, g))>\frac{q}{q-1} \max \{v(\Delta(f)), v(\Delta(g))\} .
$$

From (2), (3), (4) and Lemma 1, we have

$$
v(\alpha-\beta)>\max _{2 \leq i \leq q}\left\{v\left(\alpha-\alpha_{i}\right), v\left(\beta-\beta_{i}\right)\right\} .
$$

By using (5) and Krasner's Lemma one obtains $K(\alpha)=K(\beta)$. It is clear that $h_{K, \alpha, \beta}$ and $h_{K, \beta, \alpha}$ are bijections, inverse to each other. To prove that the above functions 
preserve the distances, it is enough to show that $\left|\alpha-\alpha_{i}\right|=\left|\beta-\beta_{i}\right|$, for any $2 \leq i \leq$ $q$. Because $|\alpha-\beta|=\left|\alpha_{i}-\beta_{i}\right|<\min \left\{\left|\alpha-\beta_{i}\right|,\left|\alpha_{i}-\beta\right|\right\}$, one has $\left|\beta-\beta_{i}\right|=\left|\alpha-\beta_{i}\right|=$ $\left|\alpha-\alpha_{i}\right|$, for any $2 \leq i \leq q$, so $h_{K, \alpha, \beta}$ and $h_{K, \beta, \alpha}$ are isometries.

We state the above result in the following theorem.

THEOREM 2. Let $q$ be a prime number, and let $f(X)$ and $g(X)$ be monic polynomials with coefficients in $K$ of degree $q$, irreducible over $K$, such that $\left(\frac{f}{g}\right)=1$. Choose a root $\alpha$ of $f(X)$ in $\bar{K}$ and let $\beta$ be the unique root of $g(X)$ in $\bar{K}$, which satisfies (2). Then $\alpha \sim_{K} \beta$.

3. Special transformations. Here and in what follows, we denote the equivalence class of an element $U \in \mathbf{C}_{p}$ by $[U]_{K}$. Thus, $[U]_{K}=[V]_{K}$ if and only if $U \sim_{K} V$. Let $\gamma=\left(\begin{array}{ll}a & b \\ c & d\end{array}\right)$ be an element of $G L_{2}(K)$. Let us consider the fractional linear transformation $z \rightarrow \gamma z:=\frac{a z+b}{c z+d}$ of $\mathbf{P}^{1}\left(\mathbf{C}_{p}\right)=\mathbf{C}_{p} \cup\{\infty\}$. We first remark that for any element $z$ of $K$, the equivalence class $[z]_{K}$ of $z$ coincides with $K$. Here we may extend the equivalence relation $\sim_{K}$ from $\mathbf{C}_{p}$ to $\mathbf{C}_{p} \cup\{\infty\}$ by letting the point at infinity be equivalent with any element of $K$. Then $[z]_{K}=\mathbf{P}^{1}(K)$ for any $z \in K$. The above transformation $z \rightarrow \gamma z$ maps the equivalence class $\mathbf{P}^{1}(K)$ to itself. We now fix an element $U$ in $\mathbf{C}_{p} \backslash K$, and denote $V=\gamma U$. We want to see that under what circumstances $U \sim_{K} V$ ? First of all, $U$ and $V$ generate the same field extension of $K$, and hence $E_{K, U}=E_{K, V}$. Therefore, the maps $h_{K, U, V}$ and $h_{K, V, U}$ are bijections inverse to each other, and one has $U \sim_{K} V$ if and only if these maps are isometries. Choose any two elements $U^{\prime}$ and $U^{\prime \prime}$ of $C_{K}(U)$. We need to see when one has the equality

$$
\left|h_{K, U, V}\left(U^{\prime \prime}\right)-h_{K, U, V}\left(U^{\prime}\right)\right|=\left|U^{\prime}-U^{\prime \prime}\right| .
$$

Let $\sigma, \tau \in G_{K}$ be such that $U^{\prime}=\sigma(U)$ and $U^{\prime \prime}=\tau(U)$. Then, since

$$
h_{K, U, V}(\sigma(U))=\sigma(V)=\sigma(\gamma U)=\gamma \sigma(U),
$$

and similarly

$$
h_{K, U, V}(\tau(U))=\tau(V)=\tau(\gamma U)=\gamma \tau(U)
$$

equation (6) reduces to

$$
|\gamma \tau(U)-\gamma \sigma(U)|=|\tau(U)-\sigma(U)|
$$

On the other hand, using the equalities $|c \tau(U)+d|=|c \sigma(U)+d|=|c U+d|$, a straightforward calculation gives

$$
|\gamma \tau(U)-\gamma \sigma(U)|=\frac{|\operatorname{det} \gamma|}{|c U+d|^{2}} \cdot|\tau(U)-\sigma(U)| .
$$

Combining (7) with (8) we derive $U \sim_{K} V$ if and only if $|\operatorname{det} \gamma|=|c U+d|^{2}$. This provides a characterisation for the set of elements $U$, which are equivalent to their image via $\gamma$.

THEOREM 3. Let $U$ be an element of $\mathbf{C}_{p} \backslash K$ and let $\gamma=\left(\begin{array}{ll}a & b \\ c & d\end{array}\right)$ be an element of $G L_{2}(K)$. Then $\gamma U \sim_{K} U$ if and only if $|\operatorname{det} \gamma|=|c U+d|^{2}$. 
Now, let $\gamma=\left(\begin{array}{ll}a & b \\ c & d\end{array}\right) \in G L_{2}(K)$ and $T, U \in \mathbf{C}_{p}$ such that $T \sim_{K} U$ and $|c T+d|=$ $|c U+d|$. By a simple calculation we have

$$
\begin{aligned}
|\sigma(\gamma T)-\gamma T| & =|\gamma \sigma(T)-\gamma T|=\frac{|\operatorname{det} \gamma|}{|c T+d|^{2}} \cdot|\sigma(T)-T| \\
& =\frac{|\operatorname{det} \gamma|}{|c U+d|^{2}} \cdot|\sigma(U)-U|=|\sigma(\gamma U)-\gamma U| .
\end{aligned}
$$

Since $E_{K, \gamma T}=E_{K, \gamma U}$, which follows from $T \sim_{K} U$, one has $\gamma T \sim_{K} \gamma U$. We have the following proposition.

Proposition 1. Let $\gamma=\left(\begin{array}{ll}a & b \\ c & d\end{array}\right) \in G L_{2}(K)$, and let $T, U \in \mathbf{C}_{p} \backslash K$ be such that $T \sim_{K}$
$U$ and $|c T+d|=|c U+d|$. Then $\gamma T \sim_{K} \gamma U$.

4. Topological properties. Let $T$ be an element of $\mathbf{C}_{p}$. Let $\left\{U_{n}\right\}_{n \geq 1}$ be a sequence of elements of $\mathbf{C}_{p}$ such that $U_{n} \in[T]_{K}$, for any $n \geq 1$. We assume that the sequence $\left\{U_{n}\right\}_{n \geq 1}$ converges to $U \in \mathbf{C}_{p}$. We want to show that $U \in[T]_{K}$. It is enough to prove the following.

Claim: The maps $h_{K, U, T}$ and $h_{K, T, U}$ are isometries and $E_{K, T}=E_{K, U}$.

Since $U_{n} \in[T]_{K}$, for any $n \geq 1$ the maps $h_{K, U_{n}, T}$ and $h_{K, T, U_{n}}$ are isometries. On the one hand we have that $\left|\sigma\left(U_{n}\right)-U_{n}\right|=|\sigma(T)-T|$, for any $\sigma \in G_{K}$ and any $n \geq 1$. By passing to the limit one obtains $|\sigma(U)-U|=|\sigma(T)-T|$, so the first part of the claim is clear. On the other hand, to prove that $E_{K, T}=E_{K, U}$, by the Galois theory it is enough to prove that $H_{K, T}=H_{K, U}$. Let $\sigma \in H_{K, T}$. One has that $\sigma \in H_{K, U_{n}}$, for any $n \geq 1$, which means $\sigma\left(U_{n}\right)=U_{n}$ for any $n \geq 1$, so $\sigma(U)=U$. This implies $H_{K, T} \subseteq H_{K, U}$. For the reverse inclusion, let $\sigma \in H_{K, U}$. Recall that $\left|\sigma\left(U_{n}\right)-U_{n}\right|=|\sigma(T)-T|$ for any $n \geq 1$. Since $U_{n} \rightarrow U$ and $\sigma(U)=U$, we have $\sigma(T)=T$ and the claim is proved. One has the following result.

THEOREM 4. Let $K$ be a fixed finite field extension of $\mathbf{Q}_{p}$. Then all equivalence classes with respect to the equivalence relation $\sim_{K}$ are topologically closed in $\mathbf{C}_{p}$.

ACKNOWLEDGEMENTS. This work was supported by Grant PN-II-ID-PCE-2012-40376, titled 'p-Adic Analytic Functions and Distributions of Sequences'. The authors are grateful to the referee for useful comments and suggestions.

\section{REFERENCES}

1. V. Alexandru, N. Popescu and A. Zaharescu, On the closed subfields of $\mathbf{C}_{p}, J$. Number Theory 68(2)(1998), 131-150.

2. V. Alexandru, N. Popescu and A. Zaharescu, The generating degree of $\mathbf{C}_{p}$, Canad. Math. Bull. 44(1) (2001), 3-11. (2001), 13-48. $417-428$.

4. J. Ax, Zeros of polynomials over local fields - The Galois action, J. Algebra 15 (1970),

5. D. Barsky, Transformation de Cauchy $p$-adique et algèbre d'Iwasawa, Math. Ann. 232(3) (1978), 255-266. 
6. A. Ioviţă and A. Zaharescu, Completions of r.a.t.-valued fields of rational functions, J. Number Theory 50(2) (1995), 202-205. $1-61$.

7. B. Mazur and P. Swinnerton-Dyer, Arithmetic of Weil curves, Invent. Math. 25 (1974),

8. S. Sen, On automorphisms of local fields, Ann. Math. 90(2) (1969), 33-46.

9. J. T. Tate, p-divisible groups, in Proc. Conf. Local Fields, Driebergen, 1966 (Springer, Berlin, Germany, 1967) 158-183.

10. A. Zaharescu, A metric symbol for pairs of polynomials over local fields, C.R. Math. Acad. Sci. Soc. R. Can. 22(4) (2000), 147-150.

11. A. Zaharescu, Lipschitzian elements over p-adic fields, Glasgow Math. J. 47 (2005), $363-372$. 